\title{
Estimativa temporal de inundações de rios em tempo linear
}

\author{
Alice Fonseca Finger ${ }^{1}$ \\ Aline Brum Loreto ${ }^{2}$ \\ Samuel Beskow ${ }^{3}$
}

\begin{abstract}
Resumo: O número de inundações em rios tem aumentado significativamente em diversas localidades do Brasil, causando enormes prejuízos à população. Diante desse fato, o presente trabalho propõe estimar o dia em que o volume de um rio irá transbordar. Para a simulação de inundação adota-se um modelo teórico matemático onde, nas resoluções das equações que representam o modelo, propõe-se aplicar métodos numéricos, a fim de analisar qual método apresenta melhor solução, considerando o esforço computacional e a exatidão da solução. O modelo hidrológico adotado neste trabalho realiza a previsão através da solução de Burgers, porém essa solução tem uma complexidade $O\left(n^{r+1}\right)$, não indicada em termos computacionais. Mas ao utilizar métodos de passos simples para prever o dia da inundação, essa complexidade se reduz para $O(n)$. No presente trabalho, mostra-se que o modelo adotado possui solução com complexidade computacional maior do que a complexidade dos métodos numéricos adaptados ao modelo e apresenta um modelo computacional que estima o dia em que o rio irá transbordar em tempo linear. Após a análise do método numérico, realiza-se a simulação numérica de inundação considerando dados hidrológicos pertinentes à área física de determinado rio da região Sul do Rio Grande do Sul (RS).
\end{abstract}

Palavras-chave: Modelos hidrológicos. Métodos numéricos. Complexidade.

\begin{abstract}
The number of floods in rivers has increased significantly in several locations in Brazil, causing huge losses to the population. Given this fact, this work focuses on estimating the day that the volume of a river will overflow. For the simulation of flood we adopt a theoretical mathematical model, where in the resolutions of the equations which represent the model, proposes apply numerical methods in order to examine what method provides a better solution, considering the computational effort and exactly of the solution. Hydrological models adopted in this work makes the prediction by solving Burgers, however, this solution has a complexity $O\left(n^{r+1}\right)$, not indicated in computational terms. But, by using methods of simple steps to predict day of the flood, this complexity is reduced to $O(n)$. The present study shows that the model adapted solution has higher computational complexity tahn the complexity of numerical methods adapted to the model and presents a computational model that estimates the day that the river will overflow in linear time.After the analysis of the numerical method we perform numerical simulation of flood considering hydrological data pertinent to the physical area of determined river in the south region of RS.
\end{abstract}

Keywords: Hydrological models. Numerical methods. Complexity.

\section{Introdução}

O número de inundações em rios tem aumentado significativamente em diversas localidades do Brasil em decorrência do crescimento desordenado das cidades brasileiras, do aumento do número de ocupações às margens de rios e também devido a precipitações intensas com duração suficiente para ultrapassar a capacidade do volume de água do rio. Essas inundações causam enormes prejuízos econômicos, como, por exemplo, interrupção da

\footnotetext{
${ }^{1}$ Curso de Ciência da Computação, UFPel, Campus Capão do Leão - Pelotas (RS) - Brasil

\{affinger@inf.ufpel.edu.br\}

${ }^{2}$ Centro de Desenvolvimento Tecnológico - CDTec/Computação, UFPel, Campus Capão do Leão - Pelotas (RS) - Brasil

\{aline.loretodinf.ufpel.edu.br\}

${ }^{3}$ Centro de Desenvolvimento Tecnológico - CDTec/Engenharia Hídrica, UFPel, Campus Porto - Pelotas (RS) - Brasil

\{samuel.beskow@ufpel.edu.br\}
}

http://dx.doi.org/10.5335/rbca.2011.1805 
atividade econômica na área inundada, e prejuízo social, como perdas materiais e humanas [1]. A população de maior poder aquisitivo tende a habitar os locais seguros, ao contrário da população carente, que ocupa as áreas de alto risco de inundação, provocando problemas sociais que se repetem por ocasião de cada cheia na região. Quando a frequência das inundações é baixa, a população ganha confiança e ignora o risco, aumentando significativamente o investimento e a densificação nas áreas inundáveis. Em outras palavras, as áreas inundáveis tornam-se mais populosas, o que pode causar, durante uma inundação, problemas com características catastróficas.

Inundaçãos ocorrem quando há precipitações intensas com duração suficiente para ultrapassar a capacidade do volume de água do rio, alagando terras próximas. Existem medidas estruturais e não estruturais para evitar as inundações, todavia as não estruturais são as mais recomendadas e ideais no controle de inundações, pois envolvem menos investimento e não causam impactos ambientais, atuando na minimização das consequências advindas desses eventos [1].

Um modelo pode ser considerado como uma representação simplificada da realidade, auxiliando no entendimento dos processos que envolvem essa realidade. Os modelos estão sendo cada vez mais utilizados em estudos ambientais, pois ajudam a entender o impacto das mudanças no uso e cobertura da terra e prever alterações futuras no ecossistema [2].

De maneira geral, um modelo é um sistema de equações e procedimentos compostos por variáveis e parâmetros, os quais mantêm seu valor inalterado durante todo o processo estudado. Assim, um parâmetro possui o mesmo valor para todos os intervalos de tempo, o que não significa que não possa variar espacialmente. As variáveis podem também mudar ao longo do tempo em que o modelo estiver sendo executado [3].

Dentre diversos modelos teóricos de inundações de rios, adota-se o modelo teórico matemático devido a Nachbin [3], por permitir adaptações quanto ao comprimento e à extensão do rio (considerando rios mais compridos do que largos), número de subdivisões ao longo do comprimento do rio e intervalo de tempo para estimar o dia em que o rio irá transbordar devido às precipitações intensas. A estimativa temporal é obtida por meio da solução de Burgers. Porém, essa solução requer elevado custo computacional em razão do grande número de operações que devem ser efetuadas.

O presente trabalho propõe realizar a estimativa temporal através de soluções numéricas resultantes de aplicações de métodos numéricos de passos simples [4]. Além disso, é feita a análise da complexidade de cada método de passos simples, assim como para o de Burgers, confirmando que o problema de previsão do tempo de inundação de um rio pode ser resolvido com métodos que possuem complexidade de tempo polinomial. Comparando as soluções desses métodos numéricos, que exigem menos esforço computacional que o método de Burgers, sugerido no modelo teórico adotado, identifica-se qual método de passos simples melhor se aplica para um rio conforme a estabilidade. Por fim, aplicam-se os dados reais de um rio da região Sul do Rio Grande do Sul neste método, tanto para a equação conservativa quanto para a não conservativa, para estimar o dia em que ocorrerá a inundação desse rio, considerando chuvas extremamente fortes por uma semana seguida à situação normal.

Com a aplicação desses métodos numéricos de passos simples, será possível obter previsões de inundações de forma mais simples e, portanto, fornecer à defesa civil estadual e demais órgãos de competência, previsões de futuras inundações com dados mais confiáveis à região Sul do Rio Grande do Sul.

\section{Modelo Hidrológico}

O modelo hidrológico é uma das ferramentas que a ciência desenvolveu para melhor entender e representar o comportamento da bacia hidrográfica e prever condições diferentes das observadas. A simulação hidrológica é limitada pela heterogeneidade física da bacia e dos processos envolvidos, o que tem propiciado o desenvolvimento de um grande número de modelos que se diferenciam em função dos dados utilizados, da discretização, das propriedades da representação dos processos e dos objetivos a serem alcançados [5].

O modelo teórico adotado considera um rio muito mais longo do que largo, como mostra a Figura 1. Esse fato tem uma consequência muito importante no nosso processo de modelagem, pois pode-se modelar a dinâmica de ondas em rios sem considerar detalhes do escoamento em cada seção transversal.

O modelo devido a Nachbin [3] é um modelo unidimensional que considera apenas variações do escoamento médio na direção longitudinal, desprezando as variações na direção transversal. Uma das hipóteses para o modelo unidimensional é que em um corte transversal do rio a velocidade da água varia muito pouco. Nesse 


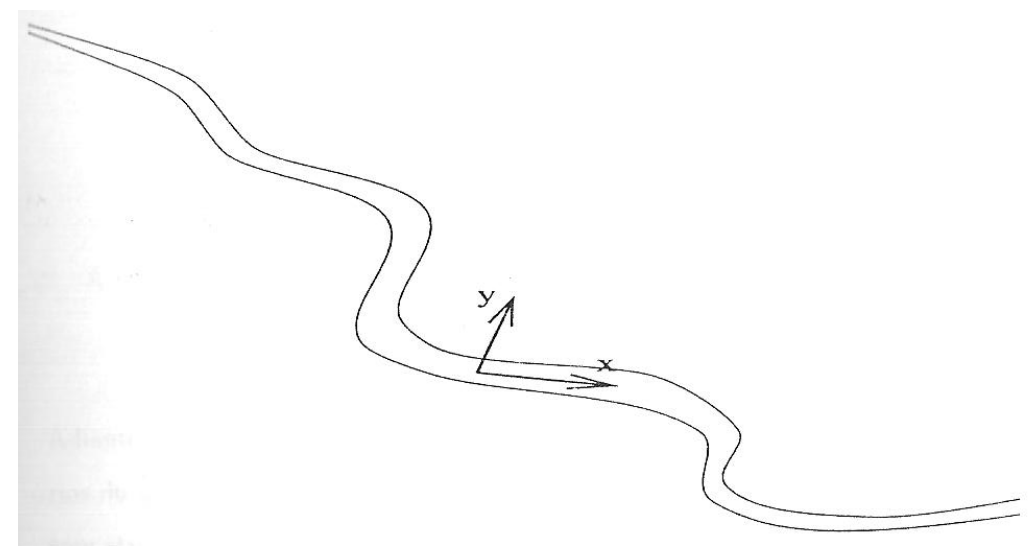

Figura 1. Vista superior do rio.

contexto, as velocidades em todos os pontos dessa seção não diferem muito da média em si. Assim, trabalhando com apenas uma velocidade, economiza-se esforço, pois manipula-se apenas uma grandeza ao invés de várias grandezas muito parecidas. As suposições feitas só são válidas porque se considera ondas muito longas, como as provenientes de inundações originadas a montante (na região mais alta do rio, ou seja, quando se segue em direção à nascente) e as geradas por chuvas extremamente fortes. Essas ondas irão propagar em direção a cidades populosas a jusante (região mais baixa do rio, quando se segue na direção à foz), podendo causar graves problemas às cidades ribeirinhas [3].

O modelo adotado estuda grandezas médias que são funções de $x$, a coordenada ao longo do rio, e do tempo $t$. As duas grandezas médias de interesse são a altura da superfície da água $h(x, t)$ e a velocidade média do escoamento $U(x, t)$ através de uma seção transversal do rio naquele ponto $x$. A seção transversal de água $S(x, t)$ é definida como a área molhada ao cortar o rio transversalmente por um plano, e o escoamento total através de uma seção transversal, por unidade de tempo, denotado por $Q(x, t)$ (Figura 2). Em outras palavras, Q é a vazão, a taxa de variação do volume no tempo. A altura da superfície livre é determinada de forma única pela função $S(x, t)$ em cada seção, já que é função crescente da outra.

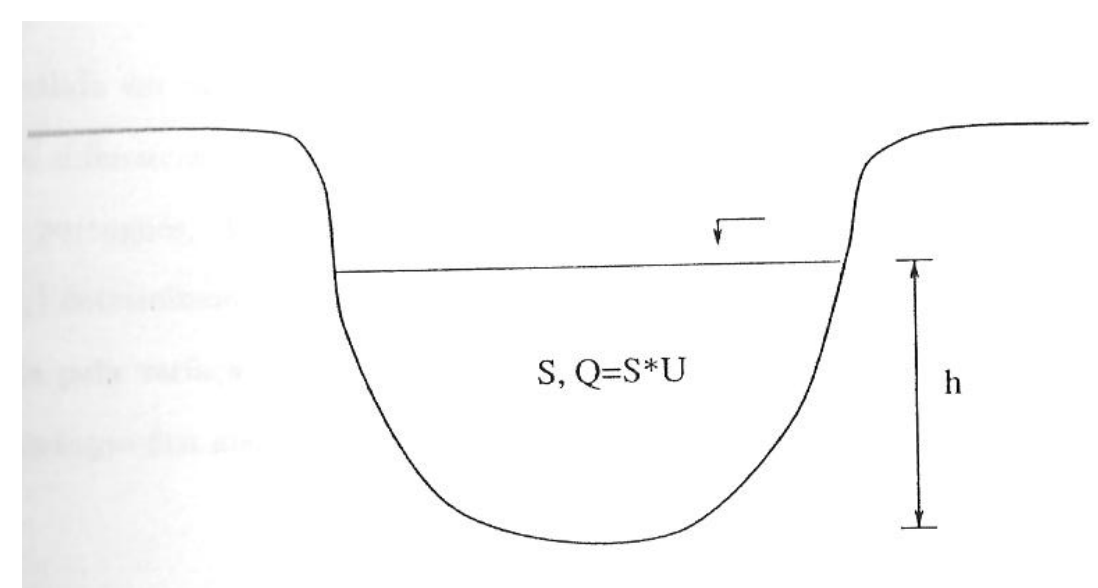

Figura 2. Seção transversal do rio.

O modelo teórico matemático devido a Nachbin [3], que dinamiza ondas em rios, além de considerar um rio muito mais longo do que largo, respeita fatores como chuvas extremamente fortes por uma semana seguida à situação normal e grandezas médias como comprimento (x), largura (y), tempo (t) e superfície (S). É importante salientar que chuvas locais, evaporação, infiltração de água no solo e as variações no escoamento em cada secção transversal são desprezadas nesse modelo, deixando a análise mais simples.

O sistema matemático de duas equações e duas incógnitas dependentes, o qual representa como as variações 
do escoamento médio de água influenciam na variação da área da seção transversal, é descrito abaixo:

$$
\begin{gathered}
S_{t}(x, t)=-Q_{x}(x, t) \\
Q=\frac{S^{2}}{2}
\end{gathered}
$$

onde

$\mathrm{Q}(\mathrm{x}, \mathrm{t})$ é a taxa de variação do volume no tempo;

$\mathrm{S}(\mathrm{x}, \mathrm{t})$ é a altura da superfície livre em cada seção transversal.

A equação diferencial (1) é obtida a partir do princípio de conservação de massa entre as seções $x_{1}$ e $x_{2} \mathrm{e}$ pela Lei Hidrológica (equação(2)).

Através de aproximações em $S_{x}$ e $S_{t}$ na equação (1) e avaliações no ponto $x_{j}$ e no instante $t_{n}$ é encontrada a seguinte equação de diferenças (forma não-conservativa da massa):

$$
S_{j}^{n+1}=S_{j}^{n}-S_{j}^{n} \frac{\Delta t}{\Delta x}\left(S_{j}^{n}-S_{j-1}^{n}\right)
$$

a qual é discretizada obtendo-se a equação de diferenças (4), considerada de forma conservativa:

$$
S_{j}^{n+1}=S_{j}^{n}-\frac{\Delta t}{2 \Delta x}\left(\left(S_{j}^{n}\right)^{2}-\left(S_{j-1}^{n}\right)^{2}\right)
$$

O presente trabalho propõe-se resolver, através de métodos numéricos de passos simples, as equações (3) e (4), mantendo-se dados descritos no modelo [3], como o comprimento do rio igual a 5 centenas de km e a superfície inicial plana e sem ondas igual a 0,3 centenas de $\mathrm{km}^{2}$. Além disso, as superfícies que supõem chuvas torrenciais por uma semana são seguidas da situação normal $(\mathrm{S}(\mathrm{x}, 0))$ :

$$
\begin{gathered}
S_{j}^{n}=y_{n}=0,3+0,1\left(1-\cos \left(\frac{2 \pi}{7}\right) t\right) \\
S_{j}^{n+1}=y_{n+1}
\end{gathered}
$$

O modelo teórico para previsão de inundação de um rio devido a Nachbin [3] propõe solução para as equações não-conservativa e conservativa através do método de Burgers. Nesta solução o volume total é, por definição, a integral de $S$ ao longo do rio. O cálculo aproximado do volume é realizado somando-se todos os $S_{j}$ 's vezes o $\Delta x$, que é o espaçamento ou variação da posição conforme o número de pontos em que o rio é seccionado para efetuar o cálculo da área da secção transversal.

\section{Análise da Complexidade dos Métodos de Passos Simples e de Burgers}

O termo "complexidade", no contexto de algoritmos, refere-se aos requerimentos de recursos necessários para que um algoritmo possa resolver um problema sob o ponto de vista computacional, ou seja, à quantidade de trabalho despendido pelo algoritmo [6]. Quando o recurso é o tempo, são escolhidas uma ou mais operações fundamentais; então, são contados os números de execuções desta operação fundamental na execução do algoritmo. Segundo Toscani [6], a escolha de uma operação como operação fundamental é aceitável se o número de operações executadas pelo algoritmo é proporcional ao número de execuções da operação fundamental.

A complexidade também pode ser vista como uma propriedade do problema, o que significa dar uma medida independentemente do tratamento dado ao problema, independentemente do caminho percorrido na busca da solução, portanto independente de algoritmos [7]. 
Do ponto de vista computacional, Garey e Johnson [8] descrevem informalmente um problema como uma questão genérica a ser respondida, geralmente possuindo vários parâmetros, ou variáveis livres.

Um problema é chamado de problema computável se existir um procedimento efetivo que o resolva em um número finito de passos, ou seja, se existe um algoritmo que leve à sua solução. Observa-se, contudo, que um problema considerado "em princípio"computável pode não ser tratável na prática, devido às limitações dos recursos computacionais para executar o algoritmo implementado [6]. Se existe um algoritmo de tempo polinomial que resolve todas as instâncias de um problema, este problema é tratável; em caso contrário, diz-se que é intratável $[6]$.

Se a complexidade é tomada como a máxima para qualquer entrada de um dado "tamanho", a complexidade é chamada "complexidade no pior caso" ou simplesmente "complexidade". Se, entretanto, é levada em conta a probabilidade de ocorrência de cada entrada de um mesmo "tamanho", a complexidade é chamada "complexidade esperada" ou "complexidade média".

A análise de um algoritmo tem como objetivo melhorar, se possível, seu desempenho e escolher entre os algoritmos disponíveis o melhor. Existem vários critérios de avaliação de um algoritmo, como quantidade de trabalho requerido, quantidade de escopo requerido, simplicidade, exatidão de resposta e otimalidade. É, entretanto, a complexidade no pior caso o critério de avaliação mais utilizado [6].

A quantidade de trabalho requerido por um algoritmo não pode ser descrita simplesmente por um número, porque o número de operações básicas efetuadas em geral não é o mesmo para qualquer entrada, depende do tamanho da entrada. Mesmo para entradas de mesmo tamanho, o número de operações efetuadas pelo algoritmo pode depender de uma entrada particular. A expressão "quantidade de trabalho requerido" também é chamada "complexidade do algoritmo" [6].

Existem vários aspectos a considerar no cálculo da complexidade de tempo de um algoritmo. Um critério pode ser medir o tempo de execução requerido por diferentes algoritmos para a solução de um problema particular em um computador particular. Entretanto, uma medida empírica é fortemente dependente tanto do programa como da máquina usada para implementar o algoritmo. Assim, uma pequena mudança no programa pode não representar uma mudança significativa no algoritmo; apesar disso, a velocidade de execução pode ser afetada. Além disso, se dois programas são comparados primeiro numa máquina e depois, em outra, as comparações podem dar resultados diferentes. Então, apesar da comparação de programas reais, rodando em computadores reais, ser uma fonte de informação importante, os resultados são, inevitavelmente, afetados pela programação e as características de máquina. Uma alternativa útil é uma análise matemática das dificuldades intrínsecas da resolução do problema computacionalmente, a qual permite muitas vezes antecipar o cálculo da complexidade para a fase de projeto do algoritmo, afetando o resultado final.

No presente trabalho o foco é a análise da complexidade dos algoritmos dos métodos numéricos de passos simples - Euler, Runge-Kutta de $2^{a}, 3^{a}$ e $4^{a}$ ordens e do algoritmo proposto por Nachbin [3], elaborado para resolver as equações não-conservativa (3) e conservativa (4). Essas equações são resolvidas através do método de Burgers, o que requer cálculo da matriz inversa, operação com elevado custo computacional.

\subsection{Complexidade dos métodos numéricos}

Como um dos objetivos do trabalho é aplicar métodos numéricos com baixa ordem de complexidade para estimar o tempo de inundação de rios, analisa-se a complexidade de cada método numérico de passos simples e do método de Burgers, descrevendo as operações fundamentais e as ordens de complexidade.

O algoritmo do método de Euler está descrito em [9]. Já os algoritmos de Runge-Kutta de $2^{a}, 3^{a}$ e $4^{a}$ ordens encontram-se em [10].

Método de Euler: o algoritmo começa com uma entrade de quatro variáveis fixas $(f, a, b, h)$ e $n$, que será o tamanho da entrada. Tomam-se como operações fundamentais a atribuição (<-) e a adição (+); logo, a complexidade nessa linha é constante. Verifica-se que o laço é executado $\mathrm{n}$ vezes, fazendo $\mathrm{n}$ atribuições.

Com isso, afirma-se que a complexidade do algoritmo do método de Euler é de ordem linear, $O(n)$.

Métodos de Runge-Kutta de $2^{a}$, $3^{a}$ e $4^{a}$ ordens: nesses algoritmos tomam-se como operações fundamentais a atribuição (<-), multiplicação (*), divisão (/) e a adição (+) e, como tamanho da entrada, o número de valores 
para $k$. Verifica-se que eles executam $n$ vezes o laço.

Assim, verifica-se que a complexidade dos algoritmos para Runge-Kutta de $2^{a}, 3^{a}$ e $4^{a}$ ordens é de ordem linear, $O(n)$.

Método de Burgers: analisa-se a complexidade computacional dos algoritmos propostos por Nachbin [3] para solução das equações não-conservativa (chamado Método Não-conservativo) e para a conservativa (chamado Método Conservativo). Estas equações foram resolvidas pelo método de Burgers, o qual requer cálculo da matriz inversa.

O algoritmo começa com atribuições para as variáveis de inicialização e com algumas dessas variáveis é definido o tamanho da matriz (a partir do tamanho da entrada). Tomam-se como operações fundamentais a adição (+), multiplicação (*), subtração (-) e comparação e, como tamanho da entrada, o número $n$ de tempos em dias e $N$ o número de pontos a ser dividido o comprimento do rio, dados necessários e iniciais para calcular o volume do rio. Verificam-se duas comparações executadas $i t+1$ vezes, onde $i t$ é o contador do primeiro laço de iteração. No laço de iteração interno tem-se complexidade $O\left(n^{r}\right)$ (complexidade polinomial de ordem $r$ ), pois o tamanho do problema decresce de uma constante $c$; neste caso, $c=-1$ e $t(n)=n-c$, onde $t(n)$ é o tamanho da entrada após a execução das operações fundamentais que antes da execução era $n$. Dessa forma, verifica-se que o algoritmo, tanto pelo método não-conservativo como para o conservativo, tem $O\left(n^{r+1}\right)$.

Tabela 1. Complexidade dos métodos numéricos e de Burgers.

\begin{tabular}{c|c|c}
\hline \hline Método & Operações Fundamentais & Ordem de Complexidade \\
\hline \hline Euler & adição e atribuição & $O(n)$ \\
\hline Runge-Kutta de $2^{a}$ ordem & adição, atribuição, multiplicação e divisão & $O(n)$ \\
\hline Runge-Kutta de $3^{a}$ ordem & adição, atribuição, multiplicação, divisão e subtração & $O(n)$ \\
\hline Runge-Kutta de $4^{a}$ ordem & adição, atribuição, multiplicação e divisão & $O(n)$ \\
\hline Burgers & adição, multiplicação, subtração e comparação & $O\left(n^{r+1}\right)$ \\
\hline \hline
\end{tabular}

Verifica-se que os métodos numéricos de passos simples propostos como solução para o problema de previsão de inundações possui em menor custo computacional, ou seja, têm ordem de complexidade computacional mais reduzida do que quando se utiliza a solução de Burgers. Algoritmos que têm ordem assintótica linear $(O(n))$ precisam de menos operações para chegar à solução, ao passo que, quando são de ordem polinomial dependente do tamanho da entrada $\left(O\left(n^{r+1}\right)\right)$, necessitam de muitas operações e um elevado custo computacional.

\section{Métodos Numéricos}

O modelo hidrológico de Nachbin [3] propõe solução da previsão do tempo de inundação através de Burgers, porém essa solução requer elevado custo computacional. Propomos uma solução através de métodos de passos simples, já que possuem uma complexidade menor do que a encontrada pela solução de Burgers. A seguir são apresentadas as equações que foram adaptadas aos métodos de passos simples escolhidos pela sua complexidade linear. As equações (3) e (4) foram adaptadas aos dados iniciais do modelo em estudo para cumprir a condição de $S_{j}^{n} \frac{\Delta t}{\Delta x}<1$ (método estável), tais como: $\epsilon=10^{-1}, \Delta x=1,25, \Delta t=1 \mathrm{e} L=5$, onde $\epsilon$ é a precisão, $\Delta x$ é a variação da posição, $\Delta t$ é variação do tempo e $L$ é o comprimento do rio em $k m$. Juntamente com os dados iniciais nas soluções numéricas das equações (3) e (4), utilizam-se os métodos numéricos de Euler e Runge-Kutta de $2^{a}, 3^{a}$ e $4^{a}$ ordens, para os quais se propõem adotar as seguintes equações:

- Para a equação (3):

$$
\begin{gathered}
h=\frac{\Delta t}{\Delta x} \\
f\left(x_{n}, y_{n}\right)=-S_{j}^{n}\left(S_{j}^{n}-S_{j-1}^{n}\right)
\end{gathered}
$$

- Para a equação (4):

$$
h=-\frac{\Delta t}{2 \Delta x}
$$




$$
f_{n+1}=\left(S_{j}^{n}\right)^{2} \quad \text { e } f_{n}=\left(S_{j-1}^{n}\right)^{2}
$$

Conforme os dados iniciais do modelo teórico adotado e as atribuições adaptadas em cada método numérico de Euler, Runge Kutta de $2^{a}, 3^{a}$ e $4^{a}$ ordens [4], calcula-se o volume do rio em cada dia de chuva com o objetivo de verificar em qual dia o rio transbordará. Na Tabela 2 encontram-se os volumes calculados para cada dia, considerando um intervalo de tempo de uma semana. Os volumes foram obtidos com a aplicação dos métodos de Euler e Runge-Kutta de $2^{a}, 3^{a}$ e $4^{a}$ ordens, comparados com a solução do método de Burgers, proposto por Nachbin [3].

Tabela 2. Métodos aplicados na equação não conservativa.

\begin{tabular}{c|c|c|c|c|c}
\hline \hline \multirow{2}{*}{} & & $\begin{array}{c}\text { Runge-Kutta } \\
\text { de } 2^{a} \text { ordem }\end{array}$ & $\begin{array}{c}\text { Runge-Kutta } \\
\text { de } 3^{a} \text { ordem }\end{array}$ & $\begin{array}{c}\text { Runge-Kutta } \\
\text { de } 4^{a} \text { ordem }\end{array}$ \\
\cline { 2 - 6 } & $\begin{array}{c}\text { Volume } \\
\mathrm{Km}^{3}\end{array}$ & $\begin{array}{c}\text { Volume } \\
\mathrm{Km}^{3}\end{array}$ & $\begin{array}{c}\text { Volume } \\
\mathrm{Km}^{3}\end{array}$ & $\begin{array}{c}\text { Volume } \\
\mathrm{Km}^{3}\end{array}$ & $\begin{array}{c}\text { Volume } \\
\mathrm{Km}^{3}\end{array}$ \\
\hline \hline 1 & 1.506 & 1.675 & 1.683 & 1.686 & 1.708 \\
\hline 2 & 1.533 & 2.075 & 2.083 & 2.118 & 2.118 \\
\hline 3 & 1.595 & 2.417 & 2.418 & 2.454 & 2.466 \\
\hline 4 & 1.763 & 2.450 & 2.436 & 2.438 & 2.497 \\
\hline 5 & 1.761 & 2.139 & 2.123 & 2.086 & 2.179 \\
\hline 6 & 1.760 & 1.716 & 1.715 & 1.665 & 1.748 \\
\hline 7 & 1.734 & 1.511 & 1.519 & 1.487 & 1.540 \\
\hline 8 & 1.673 & 1.675 & 1.683 & 1.686 & 1.708 \\
\hline \hline
\end{tabular}

Analisando a Tabela 2, verifica-se que a inundação ocorrerá aproximadamente no quarto dia de chuva por apresentar ocorrência de maior volume neste dia em todos os métodos numéricos de passos simples, coincidindo com o resultado obtido pelo método de Burgers (proposto no modelo teórico adotado).

Com interesse na conservação da massa após a inundação, calculam-se os valores de volume, obtidos pela aplicação dos métodos numéricos na equação (4), os quais são apresentados na Tabela 3.

Tabela 3. Métodos aplicados na equação conservativa.

\begin{tabular}{c|c|c|c|c|c}
\hline \hline & & $\begin{array}{c}\text { Runge-Kutta } \\
\text { de } 2^{a} \text { ordem }\end{array}$ & $\begin{array}{c}\text { Runge-Kutta } \\
\text { de } 3^{a} \text { ordem }\end{array}$ & $\begin{array}{c}\text { Runge-Kutta } \\
\text { de } 4^{a} \text { ordem }\end{array}$ \\
\cline { 2 - 6 } & $\begin{array}{c}\text { Volume } \\
\mathrm{Km}^{3}\end{array}$ & $\begin{array}{c}\text { Volume } \\
\mathrm{Km}^{3}\end{array}$ & $\begin{array}{c}\text { Volume } \\
\mathrm{Km}^{3}\end{array}$ & $\begin{array}{c}\text { Volume } \\
\mathrm{Km}^{3}\end{array}$ & $\begin{array}{c}\text { Volume } \\
\mathrm{Km}^{3}\end{array}$ \\
\hline \hline 1 & 1.692 & 1.388 & 1.742 & 1.691 & 1.143 \\
\hline 2 & 1.766 & 1.307 & 2.142 & 2.097 & 1.144 \\
\hline 3 & 1.865 & 1.676 & 2.470 & 2.441 & 1.050 \\
\hline 4 & 1.944 & 2.450 & 2.487 & 2.474 & 0.932 \\
\hline 5 & 1.967 & 2.884 & 2.177 & 2.163 & 0.880 \\
\hline 6 & 1.947 & 2.491 & 1.766 & 1.737 & 0.932 \\
\hline 7 & 1.930 & 1.800 & 1.571 & 1.526 & 1.049 \\
\hline 8 & 1.765 & 1.388 & 1.742 & 1.691 & 1.143 \\
\hline \hline
\end{tabular}

Pela Tabela 3 verifica-se que o rio transbordará no quarto dia, se considerados os métodos de Runge-Kutta de $2^{a}$ e $3^{a}$ ordens; se considerados os valores de volume pelo método de Euler, a inundação ocorrerá no quinto dia, considerando que o método de Burgers também apresentou maior volume no quinto dia. Neste caso, estima-se que a inundação ocorrerá entre o quarto e quinto dia.

\section{Erros}

Quando resolvemos problemas utilizando computação numérica, os resultados obtidos são em geral aproximados. Ressalta-se que nenhum resultado obtido pela aplicação de métodos numéricos tem valor se não for 
conhecido o controle sobre os possíveis erros envolvidos no processo. Assim, torna-se importante medir o quão próximo este resultado obtido por meio de métodos numéricos está no valor exato. Neste caso, o valor exato considerado é o volume calculado pelo método de Burgers (solução proposta pelo modelo teórico adotado).

\subsection{Tipos de erros}

Nas aproximações entre um valor exato e sua aproximação consideram-se duas formas de avaliar o erro cometido [4]:

- Erro Absoluto $\left(E A_{x}\right)$ : diferença entre o valor exato de um número $x$ e seu valor aproximado $\bar{x}$.

$$
E A_{x}=|x-\bar{x}|
$$

- Erro Relativo $\left(E R_{x}\right)$ : razão entre o erro absoluto e o valor aproximado $\bar{x}$.

$$
E A_{x}=\frac{\left|E A_{x}\right|}{\bar{x}}
$$

\subsection{Erros calculados}

No presente trabalho, considera-se como valor exato $x$ o valor obtido da aplicação do método de Burgers (método sugerido no modelo adotado [3]) e, para o valor aproximado $\bar{x}$, o resultado proveniente da aplicação dos métodos numéricos de Euler e Runge-Kutta de $2^{a}, 3^{a}$ e $4^{a}$ ordens. Com os valores obtidos da aplicação dos métodos numéricos calculam-se o erro absoluto (EA) e o erro relativo (ER) para cada método aplicado, com exatidão $\epsilon=10^{-1}$.

A Tabela 4 apresenta os erros Absoluto e Relativo calculados para os volumes (Tabela 2) obtidos através da solução da equação não-conservativa para cada dia, considerando o período de uma semana seguida de chuvas torrenciais.

Tabela 4. Erro dos métodos aplicados na equação não-conservativa.

\begin{tabular}{c|c|c|c|c|c|c|c|c}
\hline \hline \multirow{2}{*}{} & \multicolumn{3}{|c|}{} & \multicolumn{2}{c}{$\begin{array}{c}\text { Método de Runge- } \\
\text { Kutta de } 2^{a} \text { ordem }\end{array}$} & \multicolumn{2}{c}{$\begin{array}{c}\text { Método de Runge- } \\
\text { Kutta de } 3^{a} \text { ordem }\end{array}$} & \multicolumn{2}{c}{$\begin{array}{c}\text { Método de Runge- } \\
\text { Kutta de } 4^{a} \text { ordem }\end{array}$} \\
\cline { 2 - 10 } & EA & ER & EA & ER & EA & ER & EA & ER \\
\hline \hline 1 & 0.169 & 0.100 & 0.177 & 0.105 & 0.180 & 0.106 & 0.202 & 0.118 \\
\hline 2 & 0.542 & 0.261 & 0.550 & 0.264 & 0.585 & 0.276 & 0.585 & 0.276 \\
\hline 3 & 0.822 & 0.340 & 0.823 & 0.340 & 0.859 & 0.350 & 0.871 & 0.353 \\
\hline 4 & 0.687 & 0.280 & 0.673 & 0.276 & 0.675 & 0.276 & 0.734 & 0.293 \\
\hline 5 & 0.378 & 0.176 & 0.362 & 0.170 & 0.325 & 0.155 & 0.418 & 0.191 \\
\hline 6 & 0.044 & 0.025 & 0.045 & 0.026 & 0.095 & 0.057 & 0.012 & 0.006 \\
\hline 7 & 0.223 & 0.147 & 0.215 & 0.141 & 0.247 & 0.166 & 0.194 & 0.125 \\
\hline 8 & 0.002 & 0.001 & 0.010 & 0.005 & 0.013 & 0.007 & 0.035 & 0.020 \\
\hline \hline
\end{tabular}

Na Tabela 5 encontram-se os erros Absoluto e Relativo calculados a partir dos volumes descritos na Tabela 3, obtidos através da solução da equação conservativa, considerando o mesmo período de uma semana seguida de chuvas torrenciais. 
Tabela 5. Erro dos métodos aplicados na equação conservativa.

\begin{tabular}{c|c|c|c|c|c|c|c|c}
\hline \hline \multirow{2}{*}{} & \multicolumn{2}{|c|}{} & \multicolumn{2}{c|}{$\begin{array}{c}\text { Método de Runge- } \\
\text { Kutta de } 2^{a} \text { ordem }\end{array}$} & \multicolumn{2}{c|}{$\begin{array}{c}\text { Método de Runge- } \\
\text { Kutta de } 3^{a} \text { ordem }\end{array}$} & \multicolumn{2}{c}{$\begin{array}{c}\text { Método de Runge- } \\
\text { Kutta de } 4^{a} \text { ordem }\end{array}$} \\
\cline { 2 - 9 } & EA & ER & EA & ER & EA & ER & EA & ER \\
\hline \hline 1 & 0.304 & 0.219 & 0.050 & 0.028 & 0.001 & 0.000 & 0.549 & 0.480 \\
\hline 2 & 0.459 & 0.351 & 0.376 & 0.175 & 0.331 & 0.157 & 0.622 & 0.543 \\
\hline 3 & 0.189 & 0.112 & 0.605 & 0.244 & 0.576 & 0.235 & 0.815 & 0.776 \\
\hline 4 & 0.506 & 0.206 & 0.543 & 0.218 & 0.530 & 0.214 & 1.012 & 1.085 \\
\hline 5 & 0.917 & 0.317 & 0.210 & 0.096 & 0.196 & 0.090 & 1.087 & 1.235 \\
\hline 6 & 0.544 & 0.218 & 0.181 & 0.102 & 0.210 & 0.120 & 1.015 & 1.089 \\
\hline 7 & 0.130 & 0.072 & 0.359 & 0.228 & 0.404 & 0.264 & 0.881 & 0.839 \\
\hline 8 & 0.377 & 0.271 & 0.023 & 0.013 & 0.074 & 0.043 & 0.622 & 0.544 \\
\hline \hline
\end{tabular}

De acordo com os volumes obtidos, presentes nas Tabelas 2 e 3, a respeito da previsão do dia de inundação, e os erros calculados nas Tabelas 4 e 5, verifica-se que para a equação não-conservativa, a qual estima a inundação no quarto dia, o método de Runge-Kutta de $2^{a}$ ordem apresenta-se mais estável, gerando uma melhor precisão do volume em comparação. Para a equação conservativa, onde no quinto dia ocorrerá a inundação se considerar o método de Euler, e no quarto dia nos demais métodos numéricos, verifica-se que o método de Euler gerou valores para volume com boa precisão, apesar de os demais métodos apresentarem erros absoluto e relativo menores.

É importante ressaltar que o foco do presente trabalho é estimar o dia de inundação de um rio com precipitação intensa de chuva no decorrer de uma semana, além de considerar um modelo simples, o qual despreza algumas características que podem influenciar também no processo de precipitação-vazão, neste caso, o método de Euler para a solução da equação conservativa retornou o maior volume de água no quinto dia de chuvas.

\section{Estimando o dia de inundação de um rio do RS}

O principal objetivo do presente trabalho é definir um método numérico simples, com baixo custo computacional que a partir de um modelo teórico matemático para inundaçãos de rios estime o dia em que determinado rio irá transbordar, considerando chuvas torrenciais por uma semana. A partir de estudos e análises do modelo adotado e dos métodos numéricos de passos simples, utilizam-se os métodos de Runge-Kutta de $2^{a}$ ordem para solução da equação não-conservativa (se não houver a necessidade de garantir a conservação da massa) e o método de Euler, quando estamos interessados em preservar a massa.

Definidos os métodos e estes implementados no software de computação numérica Scilab (escolhido por ser um software livre que permite programação de cálculos numéricos, além da geração de gráficos e manipulações algébricas), escolhe-se um rio do Sul do Rio Grande do Sul para estimar, com nosso modelo computacional o dia em que o rio transbordará considerando precipitações intensas por uma semana.

O arroio Fragata, da estação Passo dos Carros, localizado na cidade de Pelotas/RS, região Sul do Rio Grande do Sul, possui $17 \mathrm{~km}$ de comprimento e 126, $8 \mathrm{~km}^{2}$ de superfície. Este rio foi escolhido por ser monitorado diariamente pela Agência Nacional de Águas (ANA) [11].

Para estimar o dia de inundação deste rio consideram-se os dados do modelo teórico para cumprir a condição de estabilidade: $S_{j}^{n} \frac{\Delta t}{\Delta x}<1, \Delta x=1,25$ e $\Delta t=1$, onde $\Delta x$ é a variação da posição e $\Delta t$ é variação do tempo, e os dados hidrológicos pertinentes à área física, tais como $L=17$ (comprimento do rio) e $n=5$ (número de pontos ao longo do rio).

A Tabela 6 apresenta os valores do volume do arroio Fragata, considerando as equações não-conservativa (resolvida pelo método de Runge-Kutta de $2^{a}$ ordem) e conservativa (pelo método de Euler). 
Tabela 6. Dados do arroio Fragata aplicados nos métodos de Runge-Kutta de $2^{a}$ ordem (não conservativo) e no método de Euler (conservativo).

\begin{tabular}{c|c|c}
\hline \hline Tempo em dias & Não Conservativo & Conservativo \\
\cline { 2 - 3 } & $\begin{array}{c}\text { Volume } \\
\mathrm{Km}^{3}\end{array}$ & $\begin{array}{c}\text { Volume } \\
\mathrm{Km}^{3}\end{array}$ \\
\hline \hline 1 & 1.43196 & 1.21819 \\
\hline 2 & 1.76831 & 1.21396 \\
\hline 3 & 2.05231 & 1.52389 \\
\hline 4 & 2.07018 & 2.08291 \\
\hline 5 & 1.80828 & 2.35359 \\
\hline 6 & 1.46382 & 2.01562 \\
\hline 7 & 1.29638 & 1.49182 \\
\hline 8 & 1.43196 & 1.21819 \\
\hline \hline
\end{tabular}

Com os resultados dos volumes calculados por dia (considerando uma semana de chuvas torrenciais), estima-se que o arroio irá transbordar no quinto dia por apresentar maior volume neste dia. Salienta-se que o modelo teórico adotado considera situação normal o volume no primeiro dia de chuva e o transbordo do rio quando ocorrer o salto hidráulico, ou seja, descontinuidade dos volumes de modo que ocorra uma onda de enchente, conforme observado na Figura 3 considerando a solução pela equação conservativa.

A seguir, na Figura 3 apresenta-se a simulação da previsão de inundação do arroio Fragata considerando uma semana de chuvas torrenciais.

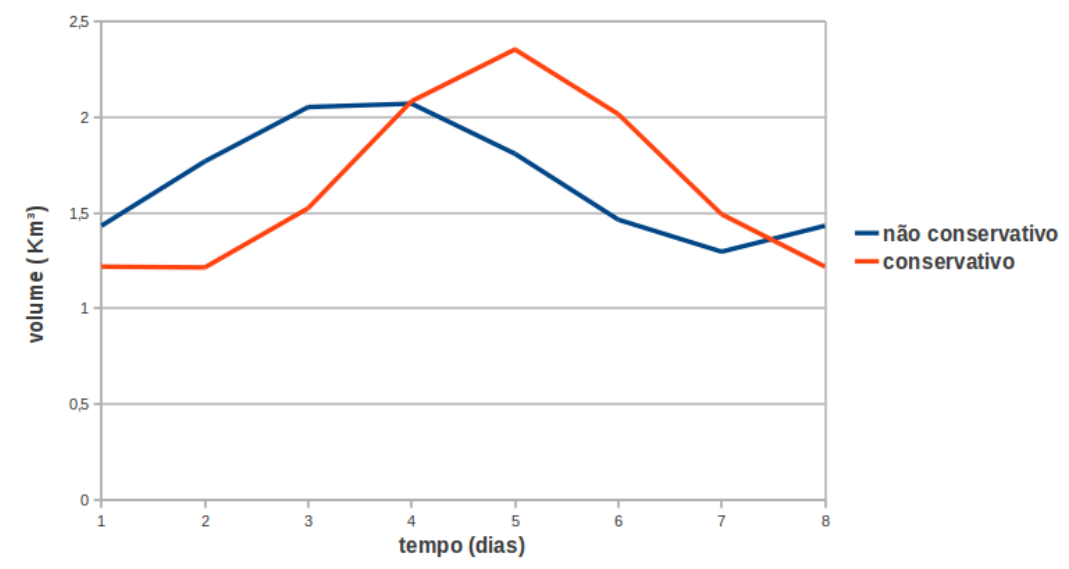

Figura 3. Gráfico previsão de inundação no Arroio Fragata.

Observa-se que as duas soluções (conservativa e não-conservativa) são muito semelhantes. De fato, elas são indistinguíveis a menos dos saltos hidráulicos. O salto hidráulico, pelo algoritmo do método conservativo, propagase mais rápido do que pelo método não-conservativo. O único efeito em usar o método na forma conservativa foi consertar a posição do salto hidráulico. Mas isso faz toda a diferença do mundo: um salto propagando mais rápido tem mais água atrás dele; é essa água que estamos perdendo no primeiro experimento com o método nãoconservativo. Do ponto de vista prático, a previsão da posição do salto é a mais importante do modelo, pois é isso que indica quando a onda de inundação atingirá cada cidade ribeirinha. Sabe-se que se deseja e se deve fazer a melhor previsão possível; por isso, é necessário escolher esquemas numéricos que estão na forma conservativa. A velocidade do salto é determinada pela lei de conservação. Se movermos a descontinuidade (salto) com a velocidade errada, água será criada ou perdida pelo modelo numérico [3].

Para certifica-se de que a estimativa temporal está correta, os volumes calculados com os dados reais foram comparados aos volumes calculados a partir das vazões deste rio, disponível no Sistema de Informações Hidrológicas - HidroWeb [11]. 
Salienta-se que a comparação leva em consideração o dia que possui o valor máximo de volume.

A Tabela 7 apresenta o volume em diferentes trechos do arroio Fragata ao longo de uma semana, dados reais utilizados para comparar e certificar a estimativa temporal de inundação do arroio Fragata.

Tabela 7. Volume $\left(\mathrm{m}^{3}\right)$ em diferentes trechos do Arroio Fragata ao longo de uma semana.

\begin{tabular}{c|c|c|c|c|c|c}
\hline \hline Tempo em dias & $\begin{array}{c}\text { Volume 1 } \\
\mathrm{m}^{3}\end{array}$ & $\begin{array}{c}\text { Volume 1-2 } \\
\mathrm{m}^{3}\end{array}$ & $\begin{array}{c}\text { Volume 2-3 } \\
\mathrm{m}^{3}\end{array}$ & $\begin{array}{c}\text { Volume 3-4 } \\
\mathrm{m}^{3}\end{array}$ & $\begin{array}{c}\text { Volume 4-5 } \\
\mathrm{m}^{3}\end{array}$ & $\begin{array}{c}\text { Volume 5-6 } \\
\mathrm{m}^{3}\end{array}$ \\
\hline \hline $10 / 09 / 2010$ & 756,17 & 6462,24 & 9215,22 & 12701,73 & 26238,10 & 95988,90 \\
\hline $11 / 09 / 2010$ & 2024,86 & 17304,38 & 24676,22 & 34012,29 & 70259,55 & 257035,98 \\
\hline $12 / 09 / 2010$ & 4458,10 & 38098,71 & 54329,16 & 74884,21 & 154689,08 & 565911,14 \\
\hline $13 / 09 / 2010$ & 12186,60 & 104146,17 & 148513,52 & 204702,55 & 422856,19 & 1546967,78 \\
\hline $14 / 09 / 2010$ & 13292,61 & 113598,14 & 161992,13 & 223280,70 & 461233,25 & 1687365,58 \\
\hline $15 / 09 / 2010$ & 4599,33 & 39305,65 & 56050,27 & 77256,49 & 159589,54 & 583838,86 \\
\hline $16 / 09 / 2010$ & 3117,26 & 26640,01 & 37988,94 & 52361,78 & 108164,28 & 395705,80 \\
\hline $17 / 09 / 2010$ & 2225,64 & 19020,27 & 27123,11 & 37384,94 & 77226,46 & 282523,58 \\
\hline \hline
\end{tabular}

Verifica-se na análise da Tabela 7 que o arroio Fragata transbordou no quinto dia, considerando uma semana de chuvas torrenciais, estimativa temporal de acordo com os resultados obtidos da aplicação dos métodos de Runge- Kutta de $2^{a}$ ordem para a equação não-conservativa e o método de Euler para a equação conservativa. $\mathrm{O}$ Sistema de Informações Hidrológicas - HidroWeb [11] disponibiliza volumes em seis trechos ao longo do arroio, porém para a nossa simulação de enchente consideramos o volume (acumulado) do último trecho 5-6; consequentemente, verifica-se o transbordamento no quinto dia de chuvas torrenciais considerando todos os trechos, ou toda a extensão do arroio. Devido aos valores dos volumes reais se apresentarem por trechos, e não único valor, houve dificuldades para realizar uma medida do erro dos resultados obtidos na simulação, problema que se pretende resolver futuramente.

\section{Conclusão}

Modelos hidrológicos geralmente são complexos e de difícil aplicação. Com a aplicação de métodos numéricos simples, deseja-se que os mesmos se tornem mais aplicáveis.

Neste trabalho adota-se um modelo teórico matemático que possibilita adequar informações físicas de determinado rio. Este modelo tem sua solução pelo método de Burgers, o que requer cálculo da matriz inversa, ou seja, custo computacional elevado. Preocupados com o tempo de processamento de tais cálculos, explora-se a solução deste modelo com métodos numéricos de passos simples: Euler e Runge-Kutta de $2^{a}, 3^{a}$ e $4^{a}$ ordens.

O crescente avanço tecnológico, que está permitindo a criação de computadores cada vez mais rápidos, pode, ingenuamente, parecer ofuscar a importância da complexidade de tempo de um algoritmo. Entretanto, acontece exatamente o inverso. Os computadores, tornando-se mais rápidos, passam a poder resolver problemas maiores, e é a complexidade do algoritmo que determina o novo tamanho máximo de problema resolvível. Para um algoritmo rápido, por exemplo de complexidade de ordem linear $O(n)$, qualquer melhoria na velocidade de execução das operações básicas é sentida e o conjunto de problemas resolvíveis por ele aumenta sensivelmente.

Para escolher qual dos métodos de passos simples retornava resultados mais aproximados, daqueles obtidos com o método de Burgers, consideram-se os mesmos dados iniciais do modelo adotado, calculam-se os erros absoluto (EA) e relativo (ER) e realiza-se uma análise sobre o dia de inundação e o respectivo método que apresentou menor erro naquele dia. Definido o método numérico de passos simples, constrói-se o modelo computacional implementado no ambiente Scilab para a solução do modelo teórico matemático adotado, porém com método numérico mais simples e com baixo custo computacional. Neste modelo computacional realiza-se a simulação para estimar o dia de inundação do arroio Fragata, da estação Passo dos Carros, localizado na cidade de Pelotas/RS, região Sul do Rio Grande do Sul.

O arroio Fragata possui $17 \mathrm{~km}$ de comprimento, $126,8 \mathrm{~km}^{2}$ de superfície, e simula-se considerando cinco pontos ao longo do seu comprimento. Com os resultados dos volumes, verifica-se que o arroio irá transbordar no quinto dia, considerando uma semana com precipitações intensas suficientes para ultrapassar a capacidade do 
volume de água do rio. O tempo estimado para ocorrer a inundação coincide com as informações fornecidas pela Agência Nacional de Águas no Sistema De Informações Hidrológicas - HidroWeb.

Com a aplicação de métodos numéricos mais simples em modelos que são mais complexos, deseja-se obter previsões de forma simples e confiável, podendo alertar a defesa civil acerca do dia de inundação, evitando prejuízos sociais, como perdas materiais e humanas.

\section{Agradecimentos}

Os autores agradecem à Fapergs pelo suporte financeiro na realização do presente trabalho.

\section{Referências}

[1] SILVA, J. B. L. Modelos de previsão de inundaçãos em tempo real para o município de Nova Era - MG. Master's thesis, Universidade Federal de Viçosa, Viçosa, 2006.

[2] MEIRELLES, M. S. P., et al. Geomática: modelos e aplicações ambientais, Brasília DF. Embrapa Informação Tecnológica, 2007.

[3] NACHBIN, A. and TABAK, E. $21^{\circ}$ Colóquio Brasileiro de Matemática: equações diferenciais em modelagem matemática computacional. IMPA, Rio de Janeiro, 1997.

[4] RUGGIERO, M. A. G. and DA ROCHA LOPES, V. L. Cálculo numérico: aspectos teóricos e computacionais. Pearson Makron Books, São Paulo, 2 edition, 1996.

[5] TUCCI, C. E. M. Modelos Hidrológicos. Editora da UFRGS, Porto Alegre, 2 edition, 2005.

[6] TOSCANI, L. V., VELOSO, P. A. Complexidade de Algoritmos: análise, projetos e métodos. Sagra-Luzzato, Porto Alegre, Instituto de Informática da UFRGS, 2001.

[7] LORETO, A et al. Complexidade Computacional de Problemas de Estatística Descritiva com Entradas Intervalares. CLEI...., 2010.

[8] GAREY, M. E., JOHNSON, D. S. Computers and intractability: a guide to the theory of NP-completeness. Freeman, San Francisco, 1979.

[9] CLÁUDIO, D. M. and MARINS, J. M. Cálculo numérico Computacional: teoria e prática. Atlas, São Paulo, 3 edition, 2000.

[10] Instituto de Ciências Exatas e da Terra - Universidade Federal de Mato Grosso. Acessado em: 12 de junho de 2011.Disponível em: <http://www.ufmt.br/icet/matematica/geraldo/>.

[11] Agência Nacional de Águas - ANA, sistema de Informações Hidrológicas - HidroWeb. Acessado em: 12 de abril de 2011.Disponível em: <http://hidroweb.ana.gov.br/>. 